\title{
A 60 Years Old Man with Adenocarcinoma of Colon and Lung with an Isolated Adrenal Metastasis: A Case Report
}

\author{
Sharma $\mathrm{NK}^{1}$, Dey $\mathrm{SN}^{2}$, Rahman $\mathrm{MM}^{3}$, Islam $\mathrm{M}^{4}$, Mukthadira ${ }^{5}$, Farzana $\mathrm{MN}^{6}$, Hasan $\mathrm{R}^{7}$
}

\begin{abstract}
The incidence of synchronous colorectal and lung cancer associated with adrenal metastasis is relatively rare. We report of patient with tumour located in the right colic flexure adjoining ascending colon, mid and partly upper lobe of right lung. Pathological examination showed the colorectal and lung cancer were poorly differentiated adenocarcinoma. Right adrenal mass was adrenocortical metastatic carcinoma. Surgical treatment and postoperative adjuvant chemotherapy for the lung cancer were different from those for colorectal cancer with pulmonary metastasis. If possible, radical resection should be performed for each cancer when synchronicity is found.
\end{abstract}

CBMJ 2020 January: vol. 09 no. 01 P: 30-37

Key words: Hemoptysis, Synchronous, Adenocarcinoma etc.

\section{Introduction}

Lung cancer is the leading cause of cancer related death in both male and female ${ }^{1}$ and 70 $80 \%$ is constituted by non-small cell lung cancer (NSCLC). Long term survival of the patients are limited when there is systemic dissemination of cancer even after surgical or medical treatment. $^{1,2}$ On the other hand, colorectal cancer is a major cause of morbidity and mortality throughout the world. ${ }^{3}$ It accounts for over $9 \%$ of all cancer incidence and it is the third most common cancer worldwide and the fourth most common cause of death. ${ }^{4,5}$ Up to $20 \%$ of the patients reveal distant metastasis and presentation.

The most common sites of distant metastasis of colorectal cancer are primarily liver and lung followed by brain and bone metastases. ${ }^{6}$ Metastasis of the adrenal gland are very infrequent whereas the adrenal glands are a common site for systemic spread for lung cancer, other include breast cancer, renal cancer, melanoma and lymphoma. The adrenal metastasis seen around $10 \%$ at the time of initial diagnosis. Unilateral adrenal metastasis is frequent usually associated with diffuse systemic spread of the primary. ${ }^{7}$

The metastasis to the adrenal glands occurs in approximately $16 \%$ of patients with metastasis colorectal carcinoma. ${ }^{8}$ Solitary adrenal metastasis of colorectal and lung cancer is a comparatively rare condition. We report the case of a patient with synchronous contralateral adrenal metastasis of colorectal and lung cancer.

\section{Case Report}

A 60 years old male patient presented to the Department of Medicine with the complaint of recurrent cough with blood stained, frothy but not foul smelling. He had no evening rise of temperature, chills, rigor and breathlessness but had right sided mild chest pain. $\mathrm{He}$ also complained of loss of appetite, constipation and weight loss for same duration.

1. Dr. Netay Kumer Sharma, Assistant Professor, Department of Radiology and Imaging, Community Based Medical College Bangladesh, Mymensingh.

2. Prof. Dr. Sankar Narayan Dey, Professor Department of Radiology and Imaging, Community Based Medical College Bangladesh, Mymensingh.

3. Prof. Dr. Md. Masudur Rahman, Professor Department of Radiology and Imaging, Community Based Medical College Bangladesh, Mymensingh.

4. Dr. Mahzabeen Islam, Associate Professor, Department of Radiology and Imaging, Community Based Medical College Bangladesh, Mymensingh.

5. Dr. Mukthadira, Assistant Professor, Department of Radiology and Imaging, Community Based Medical College Bangladesh, Mymensingh.

6. Dr. Mir Naz Farzana, Medical officer, Department of Radiology and Imaging, Community Based Medical College Bangladesh, Mymensingh.

7. Dr. Rajibul Hasan, Associate Professor, Department of Anesthesiology, Community Based Medical College Bangladesh, Mymensingh.

\section{Address of correspondence}

Email: nksharma89dr@gmail.com

Cell no: +8801711962023 
But he didn't complain of nausea, vomiting, diarrhea. The complaints began before one month from the date of admission in hospital. Along with above complaints, he also developed a swelling in the right side of the abdomen lateral to the umbilicus which disappeared during bending forwards. There was no dribbling of urine, frequency, urgency, hesitancy of urination. Color of urine was normal, stool color was intermittently black. He had no history of contact with patient with Pulmonary tuberculosis (PTB) and got admitted in the Department of Medicine, Community Based Medical College Hospital Bangladesh for better management.

On general examination he was ill looking, anaemic, temp: $98^{\circ} \mathrm{F}$, weight: $40 \mathrm{Kg}$ and absence of cyanosis, jaundice, clubbing, koilonychia, leuconychia, edema, bony tenderness. No palpable lymph nodes, thyroid gland and no engorged neck vein. After completion of general and systemic examination some pathological examinations have been done.

Laboratory findings were ESR: $80 \mathrm{~mm}$ in $1^{\text {st }}$ hour, $\mathrm{Hb} \%$ : $08 \mathrm{gm} / \mathrm{dl}$. RBS: $6.0 \mathrm{mg} / \mathrm{dl}$, S.creatinine: $1.0 \mathrm{mg} / \mathrm{dl}$. S.Bilirubin: $0.7 \mathrm{mg} / \mathrm{dl}$. ECG: Within normal limit.

Chest X-ray P/A view showed well-defined large dense rounded soft tissue mass lesion in right lung occupying mid and partly upper zones involving posterior segment of upper lobe and medial segment of middle lobe attached to right lateral chest wall associated with small dense oval soft tissue shadow at right hilar region.

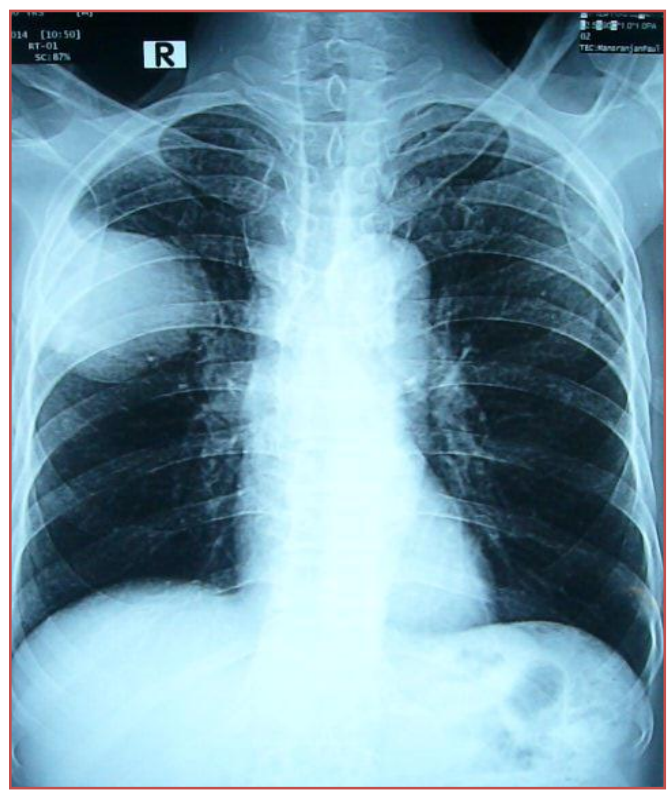

Fig 01: X-ray chest P/A view.

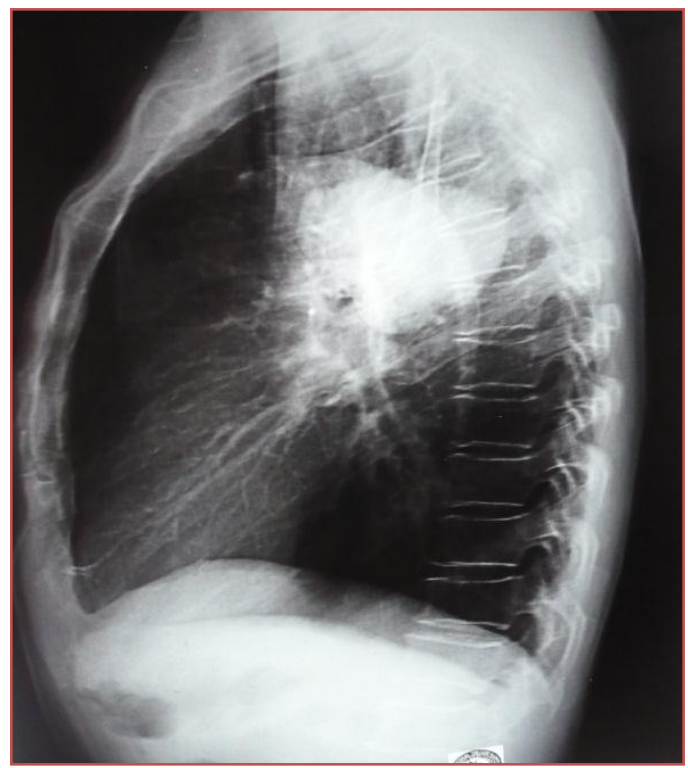

Fig 02: X-ray chest right lateral view.

Ultrasonogram (USG) of chest showed well defined soft tissue mass of size $\sim 92.6 \times 80 \mathrm{~mm}$ in lateral segment of middle lobe of right lung having no evidence of air bronchogram. Color Doppler showed increased blood flow within the mass. 


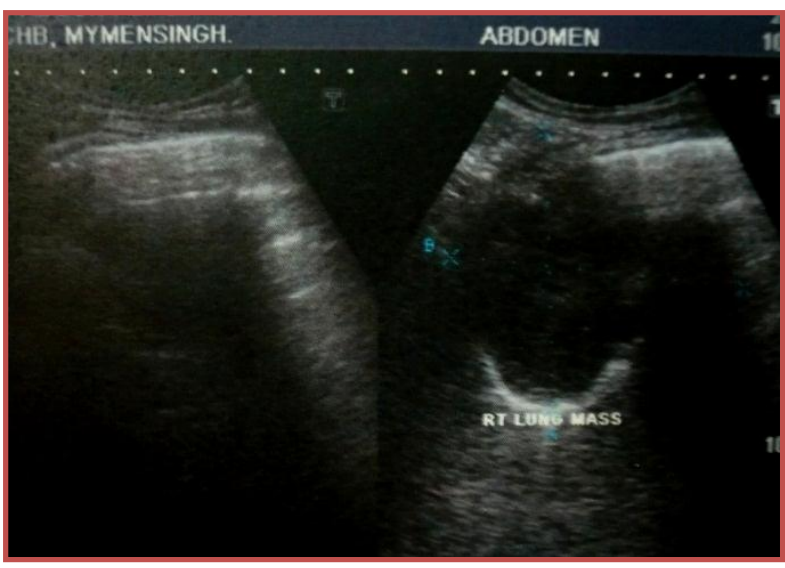

Fig 03: 2D USG of right chest.

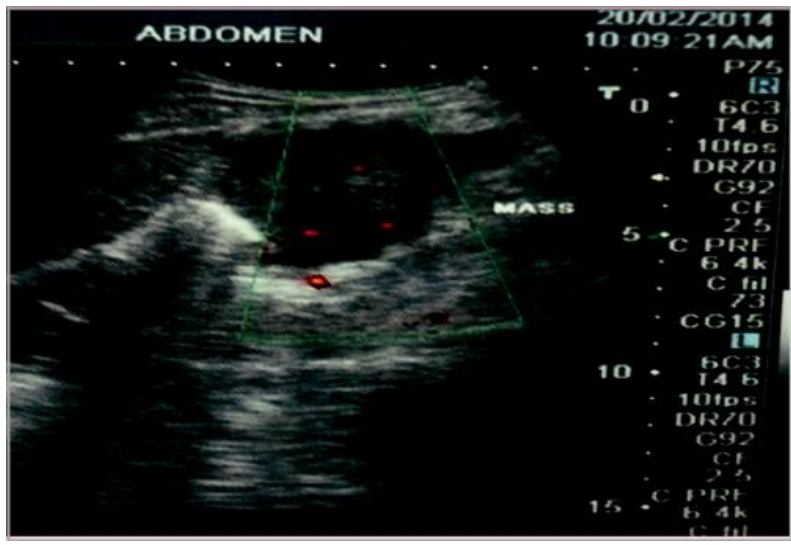

Fig 04: Color Doppler USG of right chest.

Non-contrast enhanced computed tomography (NECT) Scan of chest showed a large lobulated soft tissue mass lesion $(\sim 6.0 \times 5.8 \mathrm{~cm})$ arising from posterior segment of right upper lobe with radiating blood vessels from its margins. There is also iso-dense oval soft tissue lesion of right hilar region. There is no pleural effusion and pleural thickening.

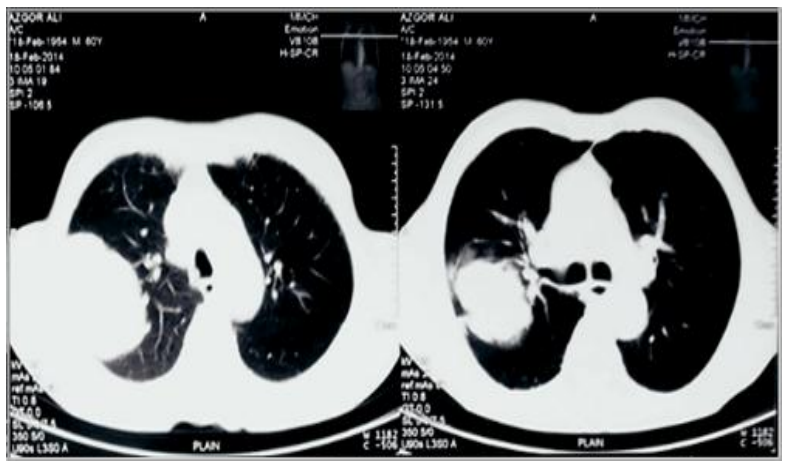

Fig 05: NECT of lung (lung window)

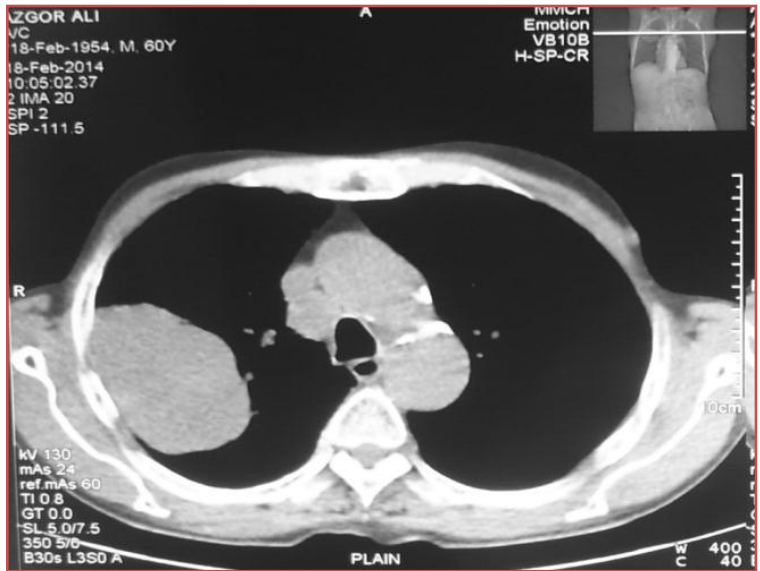

Fig 06: NECT of lung (Mediastinal window)

Contrast-enhanced computed tomography (CECT) Scan of chest showed there is moderately strong contrast enhancement of the soft tissue mass lesion arising from posterior segment of right upper lobe.
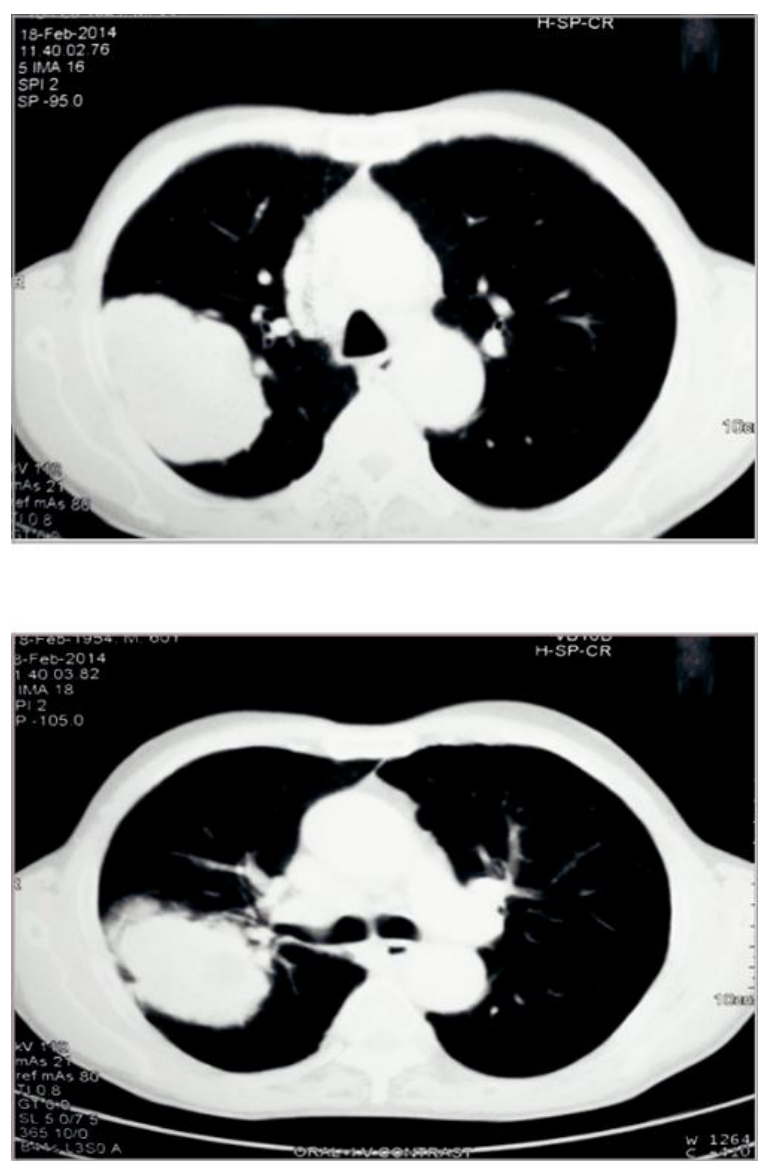

Fig 07: CECT of lung (lung window) 


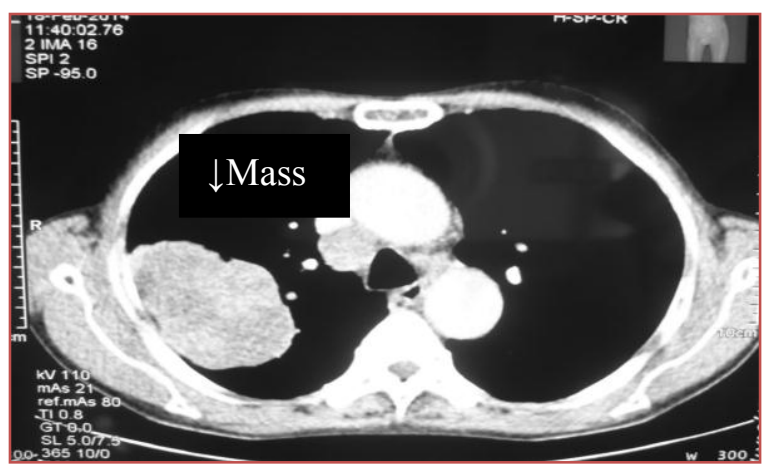

Fig 08: CECT of lung (mediastinal window)

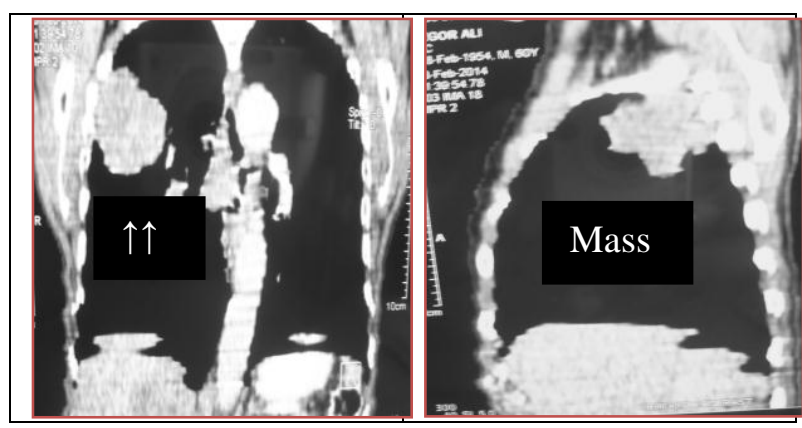

Fig 09: CECT of lung (mediastinal window with reconstruction)

X-ray Barium enema of large gut (Single contrast) There is persistent filling defect with shouldering effect of right colic flexure and adjoining ascending colon with proximal dilatation.

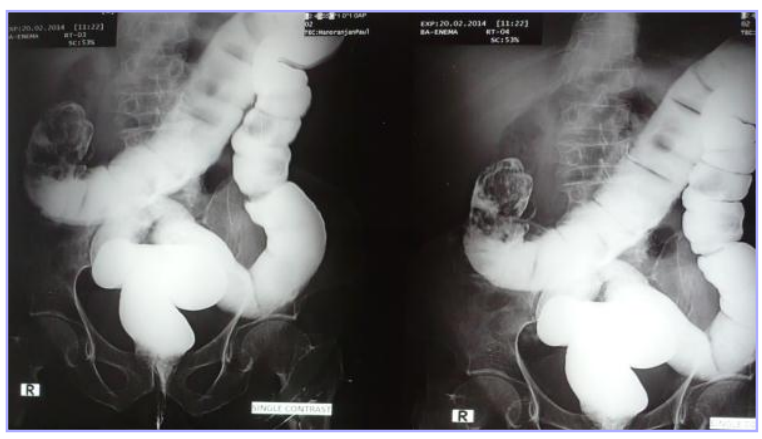

Fig 10: X-Ray barium enema (single contrast)

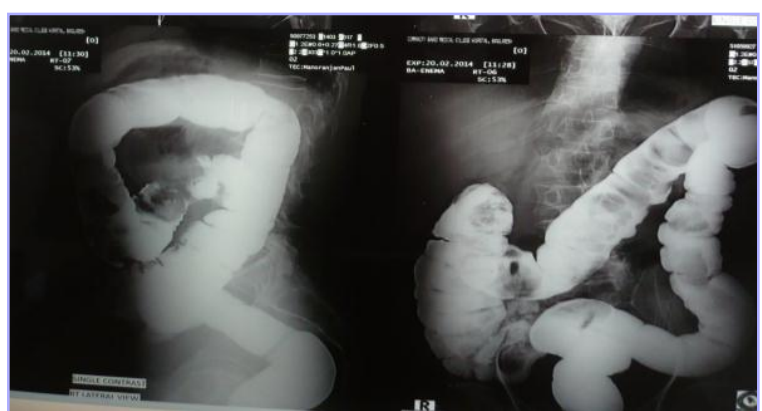

Fig 11: X-Ray barium enema (single contrast)

X-ray Barium enema of large gut (Double contrast) shouldering effect seen clearly. Possibly due to neoplastic space occupying lesions (SOL) involving right colic flexure and adjoining ascending colon.

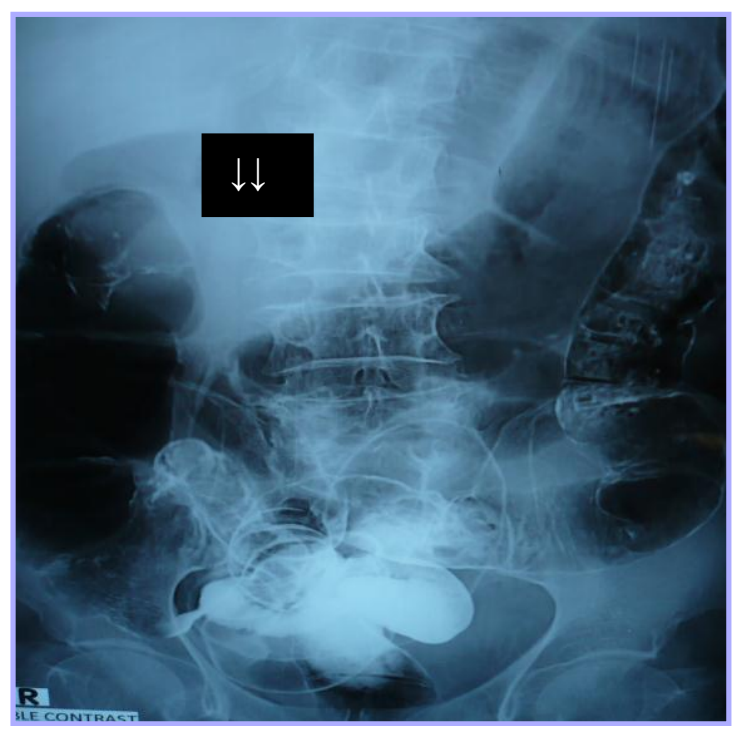

Fig 12: X-Ray barium enema (double contrast)

Abdominal ultrasonogram showed an well defined irregular hypoechoic soft tissue mass of size $\sim 57.2 \times 50.8 \mathrm{~mm}$ at right lumbar region which is merged with neighboring guts as a short segment involving ascending colon and adjoining right colic flexure. Multiple enlarged para-aortic lymph nodes seen. 


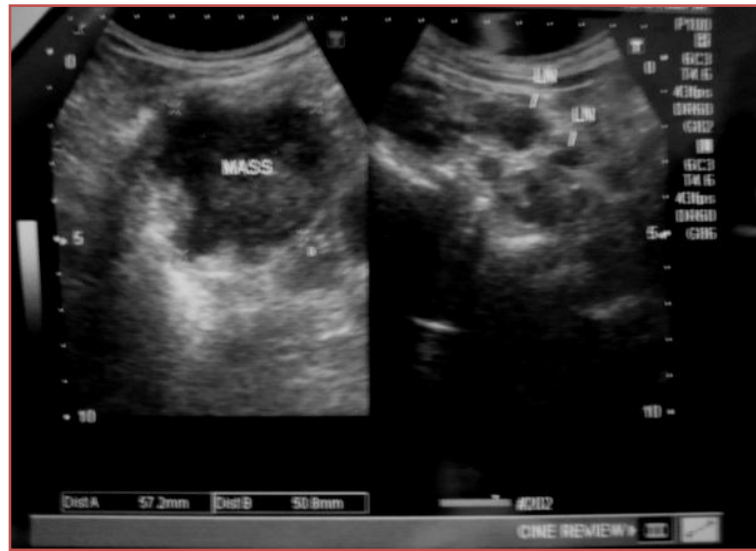

Fig 13: 2D USG of abdomen.

Another medium sized encapsulated irregular hypoechoic mass of size $~ 60.2 \times 50.1 \mathrm{~mm}$ noted over the upper pole of right kidney having few calcifications. Right kidney is free from this lesion but multiple cortical cysts seen in both kidneys.

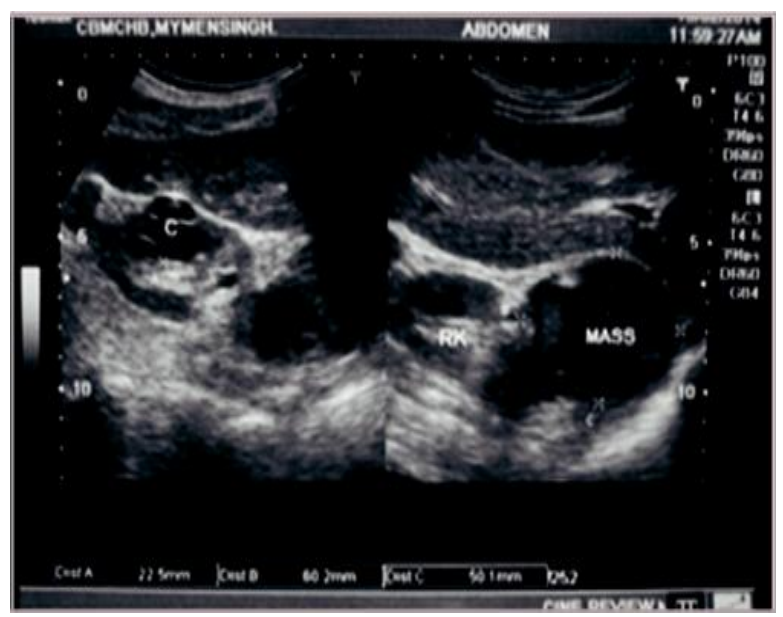

Fig 14: 2D USG of abdomen.

Color Doppler Ultrasound showed increased blood flow in the mass of colon and suprarenal gland. Diagnosis is malignant gut related mass involving ascending colon adjoining right colic flexure and metastatic mass in right suprarenal gland.
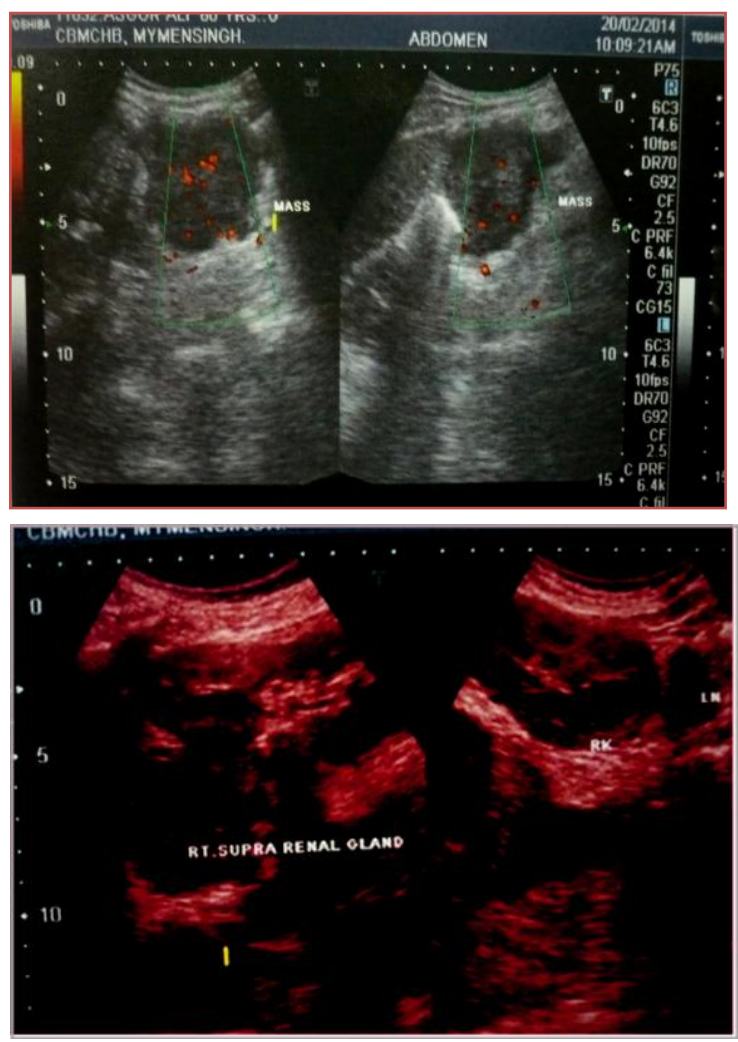

Fig 15: Color Doppler USG of abdomen.

NECT Scan of Abdomen showed there is a large iso-dense soft tissue mass lesion arising from the distal part of the ascending colon. There are small hypodense oval lesions (lymph nodes) of para-aortic, peripancreatic, mesenteric and perisplenic regions.

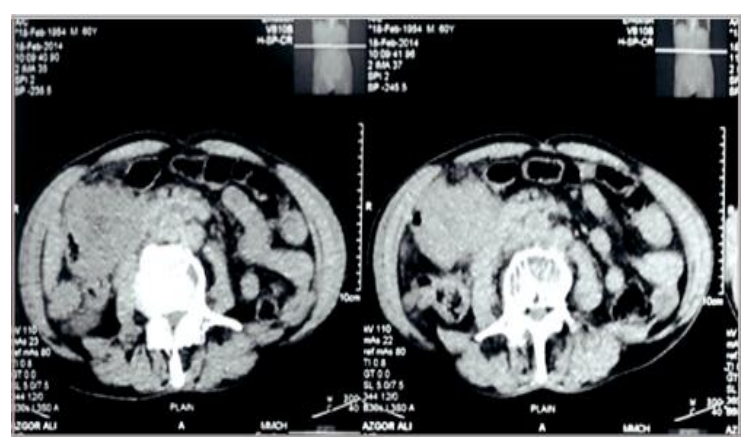

Fig 16: NECT of abdomen.

There is also large iso-dense soft tissue mass lesion noted within the right suprarenal gland. 


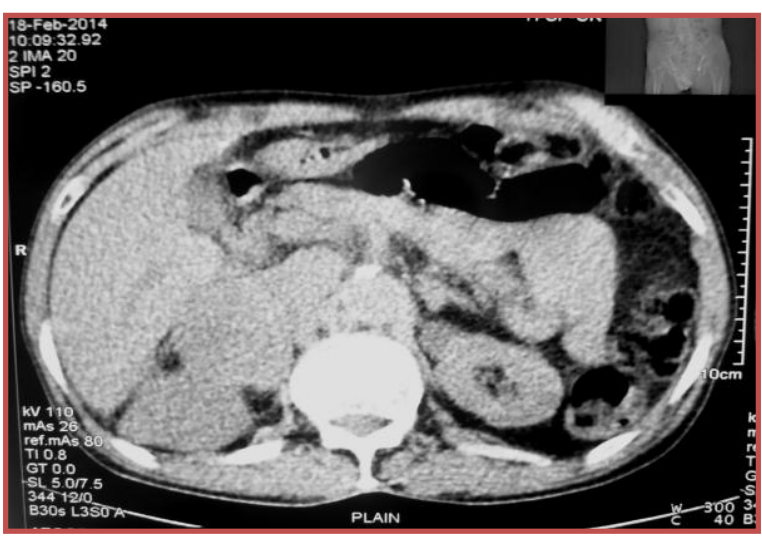

Fig 17: NECT of abdomen.

CECT Scan of Abdomen: After administration of water soluble iodinated non-ionic contrast media (Intravenous)-there is moderately strong contrast enhancement of previously mentioned lesions of ascending colon and right suprarenal gland. There are also mild contrast enhancement of mentioned oval lesions of paraaortic, peripancreatic, mesenteric and perisplenic region.
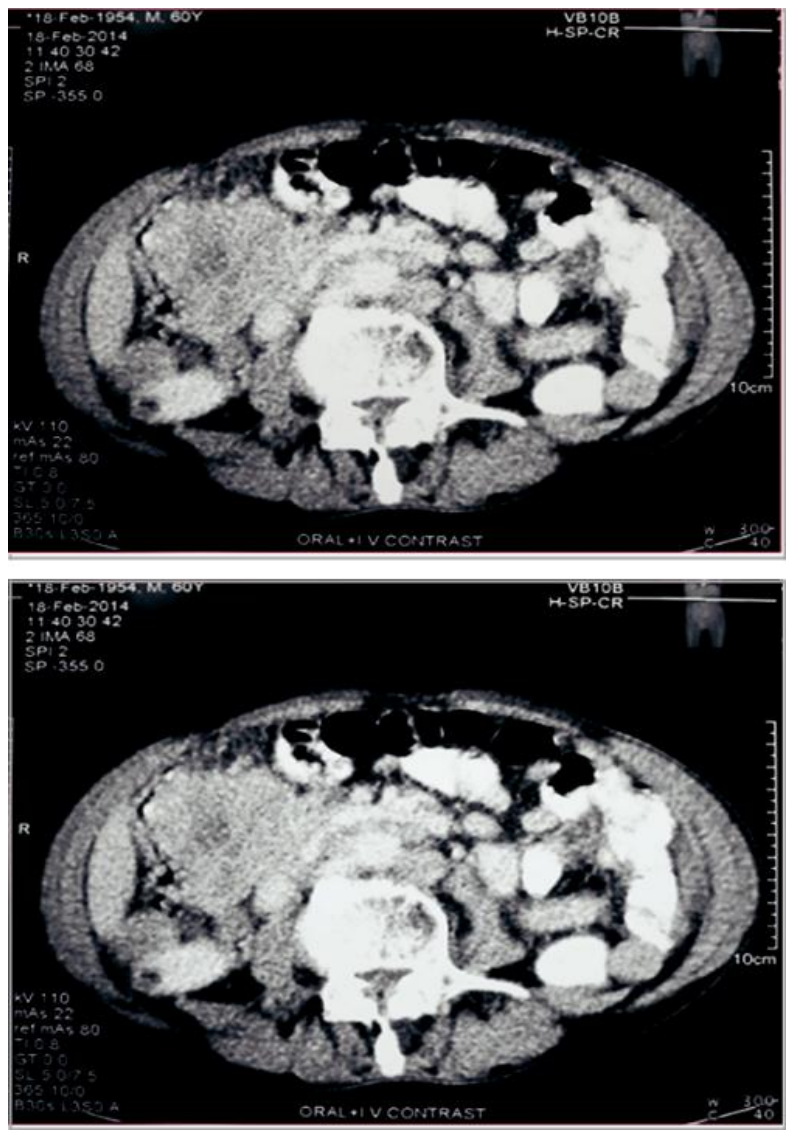

Fig 18: CECT of abdomen.
USG guided Fine needle aspiration cytology (FNAC from right lung mass, colonic and

adrenal masses): Smears showed pleomorphic cells having hyperchromatic nuclei arranged in clusters, groups, singly and forming ill-defined glands. Diagnosis of right lung mass and abdominal mass by FNAC are poorly differentiated adenocarcinoma and right adrenal mass is metastatic adrenocortical metastatic carcinoma.

\section{Discussion}

In this report, we explained the case of a male patient presented to the department of medicine with the complaints of the right sided abdominal swelling, cough with haemoptysis. During the investigations we found that his problem caused by the evidence of right sided lung cancer and cancer of right colic flexure involving partially ascending colon. Incidentally at the same time, we noticed a suspicious mass in right adrenal gland which then the pathology report supported our expectation that it was an isolated metastasis of the colonic adenocarcinoma. There are few reports on colorectal cancer with primary lung cancer as a type of multiple primary malignant neoplasm (MPMN). ${ }^{9}$

Evans et al investigated the incidence of primary lung cancer in 127281 patients with colorectal cancer and found that the incidence of primary lung cancer is $0.6 \%$ (801 cases), while the incidence of synchronous colorectal and lung cancer is much lower. The pathogenesis and biological behavior of synchronous colorectal and lung cancer vs. colorectal cancer with pulmonary metastasis are quite different, and so their treatment. Therefore, when a single solitary pulmonary lesion is found in a colorectal cancer patient, it is important to distinguish between these two possibilities for their appropriate surgical treatment and postoperative adjuvant chemotherapy.

The most common primary tumour responsible for adrenal metastasis are carcinoma of the lung, kidney and breast. Adrenal metastasis from colorectal carcinoma is less frequent and occupy the 
fourth most common site of the colorectal metastasis after lung, liver and bone. ${ }^{10}$ In literature, the global incidence of adrenal metastasis ranges between $8.6 \%$ and $27 \%$, where as the incidence of adrenal metastasis from colorectal cancer ranges from $1.9 \%$ to $17.4 \% .{ }^{11}$ Literature reveals reasonably good long term outcomes for colorectal cancers with metastasis to the liver and lung. However, the presence of metastasis to the adrenal gland is regarded as an indicator of widespread disease. The potential routes of adrenal metastasis from colorectal cancer include systemic venous, portal veins, hepatic and haematogeneous through lung. ${ }^{12}$

The presence of metastasis in the adrenal gland represents the second most frequent cause of "adrenal incidentaloma" following corticaladrenal adenomas. Although systemic therapy forms the backbone of management of metastatic colorectal cancer, the surgical resection in selected patients leads to improved survival and the early detection of the adrenal metastasis is of high importance. There is no consensus on the management of patients with isolated adrenal metastasis, the surgical resection remains controversial although a median survival of 32 months was found in the largest reported case series. ${ }^{13}$

Literature reports indicate that patients with MPMN have a better prognosis than those with a metastasis or recurrent lesion. When surgical resection combined with postoperative chemotherapy is performed for colorectal cancer patients with a secondary malignant tumour in another organ, the five years survival rate is $49 \%{ }^{14}$

Therefore, careful examination, early detection of multiple primary cancers, and active surgical approach can improve the therapeutic effects in MPMN patients with further suggestion of Immunocytochemistry, computed tomography (CT) guided FNAC of pulmonary mass and follow up positron emission tomography (PET) scan.

\section{Conclusion}

The incidence of synchronous colorectal and lung cancer is relatively low. When the first primary cancer is found, a meticulous examination of every organ should be conducted until no malignant neoplasm found. When suspicious lesions are found, further examination should be performed immediately. Synchronous radical resection should be performed when the condition of the patient permits. After radical resection, according to the pathological type and stage of the tumors and grade of metastasis, appropriate adjuvant chemotherapy should be given. The prognosis of synchronous colon and lung cancer is relatively good after careful treatment. But when adrenal metastasis occurs prognosis is very poor.

\section{References:}

1. Jett JR, Miller YE. Update in lung cancer 2005.Am J Respir Crit Care Med. 2006; 173:695-7.

2. Micke $P$, Faldum A, Metz T, Beeh KM, Bittinger $F$, Hengstler JG, et al. Staging small cell lung cancer. Veterans Administration Lung Study Group versus International Association for the Study of lung cancer-What limits limited disease? Lung Cancer. 2002;37:271-6.

3. World Health organization. Cancer Incidence in five Continents. Lyon: The World Health organization and the international Agency for Research on Cancer;2002.

4. World Cancer Research Fund and American Institutes for Cancer Research. Food, Nutrition, Physical Activity, and the prevention of Cancer: A Global perspective. Washington, DC: American Institutes for Cancer Research;2007.

5. Boyle $P$, Langman JS. $A B C$ of colorectal cancer: Epidemiology. BMJ. 2000;321:805-8.

6. Qiu M, Hu J, Yang D, Cosgrove DP, Xu R. Pattern of distant metasteses in colorerctal cancer a SEER based study. oncotarget. 2015;6:38658-66. 
7. Keranikiotis $C$, Tentes $A A$, Markakidis $S$, Vafiadis $K$. Large bilateral adrenal metastases in nonsmall cell lung cancer.World J Surg Oncol.2004;2:37.

8. Cedermark BJ, Blumension LE, Pickren JW, Holyoke DE, Elias EG. The significance of metesteses to the adrenal glands in adenocarcinoma of the colon and rectum. Surg Gynecol Obstet. 1977; 144:537-46.

9. Evans HS, Moller H, Robinson D, lewis CM, Bell CM, Hodgson SV. The risk of subsequent primary cancers after colorectal cancer in southeast England.Gut.2202;50:647-652.

10. Lam Ky, Lo CY. Metastatic tumour of the adrenal glands: A 30-year experience in a teaching hospital. clin Endocrinol (Oxf). 2002;56:95-101.

11. Murakhami S, Terakado M, Hashimoto T, Tsuji $Y$, Okubo K, Hirayama R. Adrenal metastases from rectal cancer: Report a case.Surge Today. 2003;33:126-30.

12. Katayama $A$, Mafune $K$, Makuuchi $M$. Adrenalectomy for solitary adrenal metastasis from colorectal carcinoma.Jpn.J Clin Oncol.2000;30:414-6.

13. Mourra N, Hoeffel C, Duvillard P, Guetteier C, Flejou JF, Tiret E. Adrenalectomy for clinically isolated metastasis from colorectal carcinoma: report of eight cases. Dis Colon rectum.2008;51:1846-9.

14. Tichansky DS, Cagir B, Borrazzo E, Topham A, Palazzo J, Weaver EJ, Lange A, Fry RD. Risk of second cancers in patients with colorectal carcinoids.Dis Colon rectum.2002;45:91-97. 